\title{
Letter to the Editor The FAQ initiative explaining pathology reports to patients
}

\author{
Modern Pathology (2010) 23, 1298-1300; doi:10.1038/modpathol.2010.108
}

To the Editor: Concern has been expressed by pathologists about the lack of visibility of our profession and our role as clinicians by both the general public and the medical community, despite the fact that our contribution to the care of patients with cancer and other diseases has never been greater. In an attempt to reach the goal of enhanced visibility, an international group of patient-centric pathologists organized by Juan Rosai met in Sirmione, Italy on 2-4 May 2008. One outcome of this meeting concerned the recognition that patients are becoming more active participants in their health care. Not only are they using the internet to research their disease and find specific pathologists to offer second opinion reviews, but increasingly patients are also reading their pathology reports.

No matter the clarity of the report, patients are often confused by the medical terminology they encounter in their reports. For example, in a prostate biopsy report with favorable grade adenocarcinoma on one core and high-grade prostatic intraepithelial neoplasia (PIN) on another core, I am aware of cases where patients have may focused on and worried about high-grade PIN as they read that highgrade tumors are bad. Pathologists are the best physician group to help patients better understand their reports, as many clinicians, themselves, do not fully understand pathology reports. Furthermore, based on my daily experience of discussing pathology reports with patients, clinicians are busy and often do not take the time to fully explain the report to the patient. Even if clinicians address some of the issues at the time of telling the patient about their cancer diagnosis, patients may be 'shell-shocked' and not fully integrate what they have been told. Being able to carefully review their report at home allows them to better digest the information and more meaningfully discuss the findings with their treating physicians.

Although there are several excellent websites devoted to cancer in general, as well as to organspecific cancer sites, these sites are insufficient in and of themselves. None of the sites are aimed at deciphering biopsy pathology reports on specific cancers, but rather provide information in general about various cancers.

To address these issues, a series of 'Frequently Asked Questions and Answers (FAQs)' were developed for the most common cancer biopsy reports, modeled after FAQs that the lay public is familiar with at the end of most technical instructional manuals. All the FAQs begin with the introductory statement: 'When your (prostate, colon, esophagus, etc) was biopsied, the samples taken were studied under the microscope by a specialized doctor with many years of training called a pathologist. The pathology report tells your treating doctor the diagnosis in each of the samples to help manage your care. This FAQ sheet is designed to help you understand the medical language used in the pathology report.'

It was decided that the FAQs would first be developed for biopsies as opposed to resections, as therapeutic decisions are typically driven by the biopsy report. Initially, it was also believed that it was best to concentrate on the more common cancers along with certain common biopsies performed to rule out neoplastic changes. With these guidelines in mind, FAQs were developed for the prostate, colon, esophagus, breast and lung. There are only a limited number of FAQ sheets for each organ (Table 1). The authors for the specific FAQs are listed in Table 2. The concept is that at the end of signing out a biopsy involving any one of these organs, a reference to the website can be cited.

Table 1 FAQs

Breast
1. Benign
2. Atypical hyperplasia
3. In-situ carcinoma
4. Invasive carcinoma
Colon
1. Adenomas (serrated, tubular, tubulovillous, villous) and
hyperplastic polyps
2. Adenomas with invasive carcinoma
3. Invasive adenocarcinoma not arising in an adenoma
Esophagus
1. Reflux and reactive changes
2. Barrett's with or without dysplasia
3. Invasive carcinoma (squamous cell and adenocarcinoma)
Lung
1. Carcinoma in-situ
2. Invasive carcinoma
Prostate
1. Benign prostate tissue
2. Prostate cancer on any of the cores regardless of other findings
3. Atypical, suspicious for cancer with or without high grade
prostatic intraepithelial neoplasia
4. High grade prostatic intraepithelial neoplasia without
atypical glands

1. Benign

Colon

. Adenomas with invasive carcinoma

sophagus

ysplasia

ung

1. Carcinoma in-situ

rostate

1. Benign prostate tissue

2. Prostate cancer on any of the cores regardless of other findings prostatic intraepithelial neoplasia atypical glands 
Table 2 FAQ authors

Breast

Jonathan I. Epstein, MD

The Johns Hopkins Medical Institutions, Baltimore, MD

Stuart J. Schnitt, MD

Beth Israel Deaconess Medical Center, Boston, MA

Jean F. Simpson, MD

Vanderbilt University Medical Center, Nashville, TN

Gastrointestinal system

Wendy L. Frankel, MD

Ohio State University Medical Center, Columbus, $\mathrm{OH}$

Laura W. Lamps, MD

University of Arkansas for Medical Sciences, Little Rock, AK Joel K. Greenson, MD

University of Michigan Health System, Ann Arbor, MI Elizabeth A. Montgomery

The Johns Hopkins Medical Institutions, Baltimore, MD

Lung

Jonathan I. Epstein, MD

The Johns Hopkins Medical Institutions, Baltimore, MD Jeffery L. Myers, MD

University of Michigan Health System, Ann Arbor, MI Wick, MR, MD

University of Virginia Health System, Charlottesville, VA

Yousem S.A., MD

University of Pittsburgh Medical Center, Pittsburgh, PA

\section{Prostate}

Jonathan I. Epstein, MD

The Johns Hopkins Medical Institutions, Baltimore, MD

Mahul B. Amin, MD

Cedars-Sinai Medical Center, Los Angeles, CA

Peter A. Humphrey, MD, PhD

Washington University Medical Center, St. Louis, MO

Acknowledgement: All of the FAQs were reviewed by William C. Allsbrook, Jr. MD.

The website will direct the patients to the appropriate organ and then the specific diagnosis within an organ. We would recommend adding to the end of biopsy reports the following (which can be a macro/canned text): For patients who are interested in better understanding their pathology reports, please refer to Patient FAQs (frequently asked questions), go to http://www.adasp.org and click on the tab 'FAQs for Patients'.

All FAQs have been reviewed by a small group of lay people to insure that they would be understood by the typical patient requesting his or her pathology report. As a patient requesting a pathology report will not be the 'average' patient in terms of their education and medical sophistication, the FAQs are at a somewhat more advanced level than if they were targeted for the general populace. The site-specific FAQs have also been reviewed by respective subspecialty physicians to insure that they do not infringe on the treating physicianpatient relationship.

The FAQs can be read in their entirety on-line. As an example of this process, the 'questions' for the FAQ for a biopsy report containing prostate cancer are listed in Table 3, with several 'answers' listed
Table 3 Questions for prostate cancer FAQ

1. What is 'adenocarcinoma of the prostate'?

2. What is a 'core'?

3. What is the 'Gleason grade' or 'Gleason score'?

4. What are the numbers in the Gleason score, for example $3+4=7$ or $3+3=6$ ?

5. What does it mean to have a Gleason score of 6 or 7 or $8-10$ ?

6 . What does it mean when there are different cores with different Gleason scores?

7. Does the Gleason score on my biopsy accurately indicate what the cancer grade is in the entire prostate?

8. How important is the Gleason score?

9. What does it mean if my biopsy report mentions special studies such as high molecular weight cytokeratin (HMWCK), ck903, ck5/6, p63, AMACR (racemase), 34BE12, or PIN4 cocktail?

10. What does it mean if my biopsy mentions that there is 'perineural invasion'.

11. What does it mean if in addition to cancer my biopsy report also says 'high grade prostatic intraepithelial neoplasia' or 'high grade PIN'?

12. What does it mean if in addition to cancer my biopsy report also says 'acute inflammation' (acute prostatitis) or 'chronic inflammation' (chronic prostatitis)?

13. What does it mean if my biopsy report also says 'atrophy' or 'adenosis' or 'atypical adenomatous hyperplasia' or 'seminal vesicle'?

14. What does it mean if in addition to cancer my biopsy report also says 'atypical glands' or 'atypical small acinar proliferation (ASAP)' or 'glandular atypia' or 'atypical glandular proliferation'?

below to illustrate the thought process that went into their development.

\section{What is 'adenocarcinoma of the prostate'?}

Adenocarcinoma of the prostate is a type of cancer (tumor) with a wide range of behavior from cases which are very slow growing with a low risk of causing men harm to cases which are more aggressive.

For the purposes of understanding their biopsy report, a broad definition is all that is necessary. If more in depth understanding of prostate cancer is desired, then other sources can be used.

\section{What does it mean to have a Gleason score of 6 or 7 or 8-10?}

The lowest Gleason score (least aggressive) tumor that is typically present on prostate biopsy is a 6 with higher grades (maximum Gleason score 10) corresponding to progressively more aggressive tumors.

Patients are often worried when told that they have Gleason score 6 cancer, as it is in the middle of the Gleason score range (2-10). This FAQ tells them that if they have a Gleason score 6 cancer, it is as good a grade as can be expected. Answers in some cases are purposefully left general so as to not infringe on the treating physician-patient relationship. It is not the role of the FAQ, for example, to tell the patient with 
a Gleason score 10 tumor that they have a low likelihood of surviving.

What does it mean if my biopsy report mentions special studies such as high molecular weight cytokeratin (HMWCK), ck903, ck5/6, p63, AMACR (racemase), 34BE12, or PIN4 cocktail?

These are special tests that the pathologist sometimes uses to help make the diagnosis of prostate cancer. Not all cases need these tests. Whether your report does or does not mention these tests has no bearing on the accuracy of your diagnosis.

The specifics of what the various antibodies label is not important to the patient. Patients also need to know the lack of significance if their report does not mention these tests.

What does it mean if my biopsy report also says 'atrophy' or 'adenosis' or 'atypical adenomatous hyperplasia' or 'seminal vesicle'?

All of these terms are things that the pathologist sees under the microscope that in some cases can look like cancer but are of no importance when seen on the biopsy and has nothing to do with cancer.

Pathologist often list in the report morphological findings that are of no prognostic significance and of no relevance for the patient. Synonyms are also used in the questions to account for virtually any term that a pathologist may use in their report so that the FAQs need not be individualized for specific pathologists.

\section{What does it mean if my biopsy mentions that there} is 'perineural invasion'.

The end of the answer: In some cases, it may affect treatment and in other cases it has no significance. How this finding will affect your specific treatment is best discussed with your treating doctor.

Whenever there are FAQs concerning therapy this statement is added.

As the vast majority of the FAQs deal with questions and answers that will not change over time either because they are definitional (ie, question \#1) or general in their nature (ie, question \#5), there will be relatively few FAQs that will need periodic updating. Nonetheless, it will be the role of the ADASP and the subcommittees for the organ- specific FAQs to periodically review them to update their content. Working with international pathology societies, The FAQs will also be translated into several different languages and modified if necessary for the different countries. In addition to a website containing the FAQs, they will be widely published on various pathology, clinical and patient websites.

The FAQ initiative not only helps improve patient care, as patients become more active participants in their own health care, but is also critical for pathologists to maintain and reinforce our important role in patient care. Increasingly, as many pathologists move from traditional hospital settings to laboratories, there is a risk that we will be perceived by the public and other physicians as laboratory scientists rather than as an integral component to the team involved in the diagnosis and treatment of patients. It is crucial that we as a discipline develop FAQs to explain our pathology reports. If we do not do this, other groups with less expertise, such as patient support groups or cancer societies, will eventually usurp this process from us.

Jonathan Epstein, MD The Johns Hopkins Medical Institutions, Baltimore, MD Director of the FAQ Initiative

Sponsored by the Association of Directors of Anatomic and Surgical Pathology (ADASP).

Endorsed by the College of American Pathologists (CAP).

Conceptualized by The Sirmione Group: Juan Rosai, MD (Chair), Consulenze Patologiche Oncologiche, Centro Diagnostico Italiano (CDI), Italy; Manfred Dietel, MD, Institute of Pathology Charité, Humboldt University of Medicine, Germany; Jonathan I Epstein, MD, The Johns Hopkins Medical Institutions, USA; Robert J Kurman, MD, The Johns Hopkins Medical Institutions, USA; Elizabeth Montgomery, MD, The Johns Hopkins Medical Institutions, USA; Manuel Sobrinho-Simões, MD, Medical Faculty, University of Porto, Portugal; Ronald S Weinstein, MD, University of Arizona, USA; Franco Visinoni, Milestone Medical, Bergamo, Italy.

Jonathan I Epstein

Department of Pathology, The Johns Hopkins Medical Institution, Baltimore, MD, USA E-mail: jepstein@jhmi.edu 